\title{
Holomorphic Morse inequalities and asymptotic cohomology groups: a tribute to Bernhard Riemann
}

\author{
Jean-Pierre Demailly \\ Université de Grenoble I, Département de Mathématiques \\ Institut Fourier, 38402 Saint-Martin d'Hères, France \\ e-mail: demailly@fourier.ujf-grenoble.fr
}

\begin{abstract}
The goal of this note is to present the potential relationships between certain Monge-Ampère integrals appearing in holomorphic Morse inequalities, and asymptotic cohomology estimates for tensor powers of line bundles, as recently introduced by algebraic geometers. The expected most general statements are still conjectural and owe a debt to Riemann's pioneering work, which led to the concept of Hilbert polynomials and to the Hirzebruch-Riemann-Roch formula during the XX-th century.

Résumé. Le but de cette note est de présenter les relations potentielles qui doivent exister entre certaines intégrales de Monge-Ampère et les estimations asymptotiques de cohomologie introduites récemment par les géomètres algébristes. Les énoncés les plus généraux espérés sont encore conjecturaux, et ne peuvent être formulés sans faire référence aux travaux pionniers de Riemann, qui ont conduit au concept de polynôme de Hilbert et à la formule de Hirzebruch-Riemann-Roch au cours du XXe siècle.
\end{abstract}

Mathematics Subject Classification 2010. 32F07, 14B05, 14C17

Key words. Holomorphic Morse inequalities, Monge-Ampère integrals, Dolbeault cohomology, asymptotic cohomology groups, Riemann-Roch formula, hermitian metrics, Chern curvature tensor, plurisuharmonic approximation.

Mots-clés. Inégalités de Morse holomorphes, intégrales de Monge-Ampère, cohomologie de Dolbeault, formule de Riemann-Roch, métriques hermitiennes, courbure de Chern, approximation plurisousharmonique. 


\section{Main results}

Throughout the paper, $X$ will denote a compact complex manifold and $n=\operatorname{dim}_{\mathbb{C}} X$ its complex dimension. Hirzebruch's Riemann-Roch formula [Hir54, Hir56] expresses the Euler characteristic

$$
\chi(X, \mathcal{F})=\sum_{j=0}^{n}(-1)^{j} h^{j}(X, \mathcal{F})
$$

of any coherent analytic sheaf $\mathcal{F}$ over $X$ as an explicit topological invariant computed by the integral

$$
\int_{X} \operatorname{Ch}(\mathcal{F}) \operatorname{Todd}\left(T_{X}\right)
$$

in terms of the Chern character of $\mathcal{F}$ and the Todd class of $T_{X}$. In the special case where $\mathcal{F}=\mathcal{O}\left(L^{\otimes k}\right)$ is the $k$-th tensor power of a holomorphic line bundle, the formula produces the Hilbert polynomial

$$
\chi\left(X, L^{\otimes k}\right)=P(k)
$$

which is a polynomial of degree $n$ which leading term $\frac{k^{n}}{n !} c_{1}(L)^{n}$. Moreover, if $h$ is a hermitian metric on $L$ and $\Theta_{L, h}=\frac{i}{2 \pi} D_{h}^{2}$ is the $(1,1)$ Chern curvature tensor of $(L, h)$, the top Chern class intersection number is given by

$$
c_{1}(L)^{n}=\frac{k^{n}}{n !} \int_{X} \Theta_{L, h}^{n}
$$

However, the Hilbert polynomial just gives access to the alternate sum of dimensions; in order to estimate the growth of the individual cohomology groups, it is interesting to consider appropriate "asymptotic cohomology functions". We mostly follow here notation and concepts introduced by A. Küronya [Kur06, FKL07], which extend in a natural way the concept of volume of a line bundle (cf. [DEL00], [Bou02], [Laz04]).

However, as we insist here on being able to deal with arbitrary compact complex manifolds, we are led to introduce appropriate variants of the original definitions. Recall that the Bott-Chern cohomology group $H_{\mathrm{BC}}^{p, q}(X, \mathbb{C})$ is the quotient of $d$ closed $(p, q)$-forms by $\partial \bar{\partial}$-exact $(p, q)$-forms. Then $\bigoplus_{p, q} H_{\mathrm{BC}}^{p, q}(X, \mathbb{C})$ is a bigraded finite dimensional algebra. When $X$ is Kähler, this algebra is isomorphic to the usual Hodge-De Rham cohomology algebra by the well-known $\partial \bar{\partial}$-lemma, but in general we only have canonical morphisms

$$
H_{\mathrm{BC}}^{p, q}(X, \mathbb{C}) \rightarrow H^{p, q}(X, \mathbb{C}), \quad \bigoplus_{p+q=k} H_{\mathrm{BC}}^{p, q}(X, \mathbb{C}) \rightarrow H_{\mathrm{DR}}^{k}(X, \mathbb{C})
$$

to Dolbeault and de Rham cohomology groups, which need not be isomorphisms. The Bott-Chern cohomology algebra carries a natural conjugation, and we can 
thus look at real elements $H_{\mathrm{BC}}^{p, p}(X, \mathbb{R})$ of type $(p, p)$. The first Chern class of a holomorphic line bundle $L \rightarrow X$ yields a well defined Bott-Chern class $c_{1}(L) \in H_{\mathrm{BC}}^{1,1}(X, \mathbb{R})$ and conversely, by a well known lemma due to A. Weil, such classes correspond to a holomorphic line bundle if and only if they are integral, i.e. in the image of $H^{2}(X, \mathbb{Z}) \rightarrow H^{2}(X, \mathbb{R})$ under the canonical morphism $H_{\mathrm{BC}}^{1,1}(X, \mathbb{R}) \rightarrow H^{2}(X, \mathbb{R})$.

We consider the real Neron-Severi subspace $\operatorname{NS}_{\mathbb{R}}(X) \subset H_{\mathrm{BC}}^{1,1}(X, \mathbb{R})$ generated by real combinations of all Chern classes $c_{1}(L)$ (i.e., from what we said, by integral $(1,1)$ classes $)$. Given a cohomology class $\alpha \in \mathrm{NS}_{\mathbb{R}}(X)$ there is always a sequence of $\mathbb{Q}$-line bundles $\frac{1}{k_{\nu}} L_{k_{\nu}} \in \operatorname{Pic}_{\mathbb{Q}}(X)=\operatorname{Pic}(X) \otimes_{\mathbb{Z}} \mathbb{Q}$ such that $\frac{1}{k_{\nu}} c_{1}\left(L_{k_{\nu}}\right)$ converges to $\alpha$ in $\mathrm{NS}_{\mathbb{R}}(X)$. We will simply write $\frac{1}{k} c_{1}(L) \rightarrow \alpha$ to express the fact that $\frac{1}{k} c_{1}(L)$ is close to $\alpha$ in the finite dimensional vector space $\operatorname{NS}_{\mathbb{R}}(X) \subset H_{\mathrm{BC}}^{1,1}(X, \mathbb{R})$, with its natural Hausdorff topology.

(1.5) Definition. Let $X$ be a compact complex manifold. One defines the asymptotic (analytic) q-cohomology function on $\mathrm{NS}_{\mathbb{R}}(X)$ to be

$$
\begin{aligned}
\widehat{h}^{q}(X, \alpha) & =\limsup _{k \rightarrow+\infty, \frac{1}{k} c_{1}(L) \rightarrow \alpha} \frac{n !}{k^{n}} h^{q}(X, L) \\
& =\inf _{\varepsilon>0, k_{0}>0} \sup _{k \geqslant k_{0},\left\|\frac{1}{k} c_{1}(L)-\alpha\right\| \leqslant \varepsilon} \frac{n !}{k^{n}} h^{q}(X, L) .
\end{aligned}
$$

where the pair $(k, L)$ runs over $\mathbb{N}^{*} \times \operatorname{Pic}(X)$.

From the very definition, $\widehat{h}^{q}$ is an upper semi-continuous function on $N S_{\mathbb{R}}(X)$ and it is positively homogeneous of degree $n$, namely

$$
\widehat{h}^{q}(X, \lambda \alpha)=\lambda^{n} \widehat{h}^{q}(X, \alpha)
$$

for all $\alpha \in \mathrm{NS}_{\mathbb{R}}(X)$ and all $\lambda \geqslant 0$. In the general case of compact complex manifolds, the fact that $\widehat{h}^{q}(X, \alpha)$ is finite follows from spectral theory estimates for the complex Laplace-Beltrami operators (this will become quite clear from the discussions below).

For a line bundle $L$, we simply denote $\widehat{h}^{q}(X, L)=\widehat{h}^{q}\left(X, c_{1}(L)\right)$. In this case, things become a little bit simpler, and especially, for $q=0$, one recovers the usual concept of volume of a line bundle.

(1.7) Proposition. If $X$ is projective algebraic or $q=0$, then

$$
\widehat{h}^{q}(X, L)=\limsup _{k \rightarrow+\infty} \frac{n !}{k^{n}} h^{q}\left(X, L^{\otimes k}\right)=\lim _{k \rightarrow+\infty} \frac{n !}{k^{n}} h^{q}\left(X, L^{\otimes k}\right) .
$$

Moreover, in these cases, the map $\alpha \mapsto \widehat{h}^{q}(X, \alpha)$ is (locally) Lipschitz continuous on $\mathrm{NS}_{\mathbb{R}}(X)$. 
The proof is derived from arguments quite similar to those already developed in $\left[\right.$ Kur05]. Actually, let us introduce $\operatorname{DNS}_{\mathbb{R}}(X) \subset \mathrm{NS}_{\mathbb{R}}(X)$ to be the subspace generated by classes of integral divisors $D$ on $X$ ("divisorial Neron-Severi group"). If $X$ is projective algebraic then $\operatorname{DNS}_{\mathbb{R}}(X)=\mathrm{NS}_{\mathbb{R}}(X)$, but the inclusion can be strict in general (e.g. on complex 2-tori which only have indefinite integral $(1,1)$ classes, cf. [BL04]). If $D=\sum p_{j} D_{j}$ is an integral divisor, we define its norm to be $\|D\|=\sum\left|p_{j}\right| \operatorname{Vol}_{\omega}\left(D_{j}\right)$, where the volume of an irreducible divisor is computed by means of a given hermitian metric $\omega$ on $X$; in other words, this is precisely the mass of the current of integration $[D]$ with respect to $\omega$. Clearly, since $X$ is compact, we get equivalent norms for all choices of hermitian metrics $\omega$ on $X$. We can also use $\omega$ to fix a normalized metric on $H_{\mathrm{BC}}^{1,1}(X, \mathbb{R})$. Elementary properties of potential theory show that $\left\|c_{1}(\mathcal{O}(D))\right\| \leqslant C\|D\|$ for some constant $C>0$ (but the converse inequality is of course wrong in most cases). Proposition 1.7 is a simple consequence of the following more precise cohomology estimates which will be proved in section 2 .

(1.8) Theorem. Let $X$ be a compact complex manifold. Fix a finitely generated subgroup $\Gamma$ of the group of $\mathbb{Z}$-divisors on $X$. Then there are constants $C, C^{\prime}$ depending only on $X$, its hermitian metric $\omega$ and the subgroup $\Gamma$, satisfying the following properties.

(a) Let $L$ and $L^{\prime}=L \otimes \mathcal{O}(D)$ be holomorphic line bundles on $X$, where $D \in \Gamma$ is an integral divisor. Then

$$
\left|h^{q}\left(X, L^{\prime}\right)-h^{q}(X, L)\right| \leqslant C\left(\left\|c_{1}(L)\right\|+\|D\|\right)^{n-1}\|D\| .
$$

(b) On the subspace $\operatorname{DNS}_{\mathbb{R}}(X)$, the asymptotic q-cohomology function $\widehat{h}^{q}$ satisfies a global estimate

$$
\left|\widehat{h}^{q}(X, \beta)-\widehat{h}^{q}(X, \alpha)\right| \leqslant C^{\prime}(\|\alpha\|+\|\beta\|)^{n-1}\|\beta-\alpha\| .
$$

In particular (without any further assumption on $X), \widehat{h}^{q}$ is locally Lipschitz continuous on $\operatorname{DNS}_{\mathbb{R}}(X)$.

Our ambition is to extend the function $\widehat{h}^{q}$ in a natural way to the full cohomology group $H_{\mathrm{BC}}^{1,1}(X, \mathbb{R})$. The main trouble, already when $X$ is projective algebraic, is that the Picard number $\rho(X)=\operatorname{dim}_{\mathbb{R}} \mathrm{NS}_{\mathbb{R}}(X)$ may be much smaller than $\operatorname{dim}_{\mathbb{R}} H_{\mathrm{BC}}^{1,1}(X, \mathbb{R})$, namely, there can be rather few integral classes of type $(1,1)$ on $X$. It is well known for instance that $\rho(X)=0$ for a generic complex torus a dimension $n \geqslant 2$, while $\operatorname{dim}_{\mathbb{R}} H_{\mathrm{BC}}^{1,1}(X, \mathbb{R})=n^{2}$. However, if we look at the natural morphism

$$
H_{\mathrm{BC}}^{1,1}(X, \mathbb{R}) \rightarrow H_{\mathrm{DR}}^{2}(X, \mathbb{R}) \simeq H^{2}(X, \mathbb{R})
$$

to de Rham cohomology, then $H^{2}(X, \mathbb{Q})$ is dense in $H^{2}(X, \mathbb{R})$. Therefore, given a class $\alpha \in H_{\mathrm{BC}}^{1,1}(X, \mathbb{R})$ and a smooth $d$-closed $(1,1)$-form $u$ in $\alpha$, we can find an infinite sequence $\frac{1}{k} L_{k}(k \in S \subset \mathbb{N})$ of topological $\mathbb{Q}$-line bundles, equipped 
with hermitian metrics $h_{k}$ and compatible connections $\nabla_{k}$ such that the curvature forms $\frac{1}{k} \Theta_{\nabla_{k}}$ converge to $u$. By using Kronecker's approximation with respect to the integral lattice $H^{2}(X, \mathbb{Z}) /$ torsion $\subset H^{2}(X, \mathbb{R})$, we can even achieve a fast diophantine approximation

$$
\left\|\Theta_{\nabla_{k}}-k u\right\| \leqslant C k^{-1 / b_{2}}
$$

for a suitable infinite subset $k \in S \subset \mathbb{N}$ of multipliers. Then in particular

$$
\left\|\Theta_{\nabla_{k}}^{0,2}\right\|=\left\|\Theta_{\nabla_{k}}^{0,2}-u^{0,2}\right\| \leqslant C k^{-1 / b_{2}},
$$

and we see that $\left(L_{k}, h_{k}, \nabla_{k}\right)$ is a $C^{\infty}$ hermitian line bundle which is extremely close to being holomorphic, since $\left(\nabla_{k}^{0,1}\right)^{2}=\Theta_{\nabla_{k}}^{0,2}$ is very small. We introduce the complex Laplace-Beltrami operator

$$
\bar{\square}_{k}=\left(\nabla_{k}^{0,1}\right)\left(\nabla_{k}^{0,1}\right)^{*}+\left(\nabla_{k}^{0,1}\right)^{*}\left(\nabla_{k}^{0,1}\right)
$$

and look at its eigenspaces in $L^{2}\left(X, \Lambda^{0, q} T^{\star} X \otimes L_{k}\right)$ with the metric induced by $\omega$ on $X$ and $h_{k}$ on $L_{k}$. In the holomorphic case, Hodge theory tells us that the 0-eigenspace is isomorphic to $H^{q}\left(X, \mathcal{O}\left(L_{k}\right)\right)$, but in the "almost holomorphic case" the 0-eigenvalues deviate from 0 , essentially by a shift of the order of magnitude of $\left\|\Theta_{\nabla_{k}}^{0,2}\right\| \sim k^{-1 / b_{2}}$ (see [Lae02], chapter 4). It is thus natural to introduce in this case

(1.10) Definition. Let $X$ be a compact complex manifold and $\alpha \in H_{\mathrm{BC}}^{1,1}(X, \mathbb{R})$ an arbitrary Bott-Chern $(1,1)$-class. We define the "transcendental" asymptotic $q$-cohomology function to be

$$
\widehat{h}_{\mathrm{tr}}^{q}(X, \alpha)=\inf _{u \in \alpha} \limsup _{\substack{\varepsilon \rightarrow 0, k \rightarrow+\infty, L_{k}, h_{k}, \nabla_{k}, \frac{1}{k} \Theta_{\nabla_{k}} \rightarrow u}} \frac{n !}{k^{n}} N\left(\bar{\square}_{k}, k \varepsilon\right)
$$

where the limsup runs over all 5-tuples $\left(\varepsilon, k, L_{k}, h_{k}, \nabla_{k}\right)$, and where $N\left(\bar{\square}_{k}, k \varepsilon\right)$ denotes the sum of dimensions of all eigenspaces of eigenvalues at most equal to $k \varepsilon$ for the Laplace-Beltrami operator $\bar{\square}_{k}$ associated with $\left(L_{k}, h_{k}, \nabla_{k}\right)$ and the base hermitian metric $\omega$.

The word "transcendental" refers here to the fact that we deal with classes $\alpha$ of type $(1,1)$ which are not algebraic or even analytic. Of course, in the definition, we could have restricted the limsup to families satisfying a better approximation property $\left\|\frac{1}{k} \Theta_{\nabla_{k}}-u\right\| \leqslant C k^{-1-1 / b_{2}}$ for some large constant $C$ (this would lead a priori to a smaller limsup, but there is enough stability in the parameter dependence of the spectrum for making such a change irrelevant). The minimax principle easily shows that definition 1.10 does not depend on $\omega$, as the eigenvalues are at most multiplied or divided by constants under a change of base metric. When $\alpha \in \mathrm{NS}_{\mathbb{R}}(X)$, by restricting our families $\left\{\left(\varepsilon, k, L_{k}, h_{k}, \nabla_{k}\right)\right\}$ to the case of holomorphic line bundles only, we get the obvious inequality

$$
\widehat{h}^{q}(X, \alpha) \leqslant \widehat{h}_{\mathrm{tr}}^{q}(X, \alpha), \quad \forall \alpha \in \mathrm{NS}_{\mathbb{R}}(X) .
$$


It is natural to raise the question whether this is always an equality. Hopefully, the calculation of the quantities limsup $\frac{n !}{k^{n}} N\left(\bar{\square}_{k}, k \varepsilon\right)$ is a problem of spectral theory which is completely understood since a long time. In fact, as a consequence of the techniques of [Dem85, Dem91, Lae02], one gets

(1.12) Theorem. With the above notations and assumptions, one has

$$
\limsup _{\varepsilon \rightarrow 0, k \rightarrow+\infty, L_{k}, h_{k}, \nabla_{k}, \frac{1}{k} \Theta_{\nabla_{k}} \rightarrow u} \frac{n !}{k^{n}} N\left(\bar{\square}_{k}, k \varepsilon\right)=\int_{X(u, q)}(-1)^{q} u^{n}
$$

where $X(u, q)$ is the open set of points $x \in X$ where $u(x)$ has signature $(n-q, q)$. Therefore

$$
\widehat{h}_{\mathrm{tr}}^{q}(X, \alpha)=\inf _{u \in \alpha} \int_{X(u, q)}(-1)^{q} u^{n} \quad(u \text { smooth }) .
$$

The first equality follows mainly from Theorems 2.16 and 3.14 of [Dem85], which even yield explicitly the limit for any given $\varepsilon$ outside a countable set (the limit as $\varepsilon \rightarrow 0$ is then obtained from the calculations of page 224 after Cor. 4.3). One has to observe, in the case of sequences of "almost holomorphic line bundles" considered here, that the perturbation indeed goes to 0 , and also that all constants involved in the calculations of [Dem85] are uniformly bounded; see [Dem91] and [Lae02] for more details on this. Therefore, we can reformulate more explicitly our previous question in the following terms.

(1.13) Question. For every $\alpha \in \mathrm{NS}_{\mathbb{R}}(X)$, is it true that

$$
\widehat{h}^{q}(X, \alpha)=\inf _{u \in \alpha} \int_{X(u, q)}(-1)^{q} u^{n} \quad(u \text { smooth }) ?
$$

(Note: it is known, from the holomorphic Morse inequalities proved in [Dem85], that the inequality $\leqslant$ always holds true).

In general, equality (1.13) seems rather hard to prove. In some sense, this would be an asymptotic converse of the Andreotti-Grauert theorem [AG62] : under a suitable $q$-convexity assumption, the latter asserts the vanishing of related cohomology groups in degree $q$; here, conversely, assuming a known growth of these groups in degree $q$, we expect to be able to say something about the $q$-index sets of suitable hermitian metrics on the line bundles under consideration.

For degree $q=0$, however, we deal with sections rather than with cohomology classes, and complex pluripotential theory makes things much easier. In the case $q=0$, there are for instance some well known methods to compute the volume $\operatorname{Vol}(\alpha)$ of a transcendental class $\alpha \in H_{\mathrm{BC}}^{1,1}(X, \mathbb{R})$.

(1.14) Definition. Let $X$ be a compact complex $n$-fold. We denote by

(a) $\mathcal{E}_{X} \subset H_{\mathrm{BC}}^{1,1}(X, \mathbb{R})$ the pseudoeffective cone of $X$, namely the cone of classes of closed positive $(1,1)$-currents; this is a closed convex cone; 
(b) $\mathcal{E}_{X}^{+} \subset \mathcal{E}_{X}$ the cone consisting of classes of Kähler currents, i.e. positive currents which admit a positive lower bound $T \geqslant \varepsilon \omega$ where $\omega$ is a smooth positive $(1,1)$-form on $X$ and $\varepsilon>0$; this is an open convex cone.

Given a class $\alpha \in H_{\mathrm{BC}}^{1,1}(X, \mathbb{R})$, we set $\operatorname{Vol}(\alpha)=0$ if $\alpha \notin \varepsilon_{X}^{+}$. Otherwise, if $\alpha \in \mathcal{E}_{X}^{+}$, the main approximation theorem of [Dem92] shows that the class $\alpha$ contains Kähler currents $T$ with analytic singularities, i.e. such that their local potentials $\varphi$ of $T$ have singularities of the form $\varphi=\left.\frac{1}{k} \log \left|\sum_{j}\right| g_{j, k}\right|^{2} \bmod C_{\widetilde{X}}^{\infty}$, for suitable local holomorphic functions $\left(g_{j, k}\right)$. Then there exists a blow-up $\mu: \widetilde{X} \rightarrow X$ of $X$ such that $\mu^{*} T=[E]+\beta$, where $E$ is a divisor supported on $\mu^{-1}\left(\bigcap g_{j, k}^{-1}(0)\right)$ and $\beta$ a smooth closed positive $(1,1)$-form on $\widetilde{X}$ (cf. [BDPP04]). One can define

$$
\begin{aligned}
& \operatorname{Vol}(T)=\int_{X \backslash \operatorname{sing}(T)} T^{n}=\int_{\widetilde{X}} \beta^{n}, \\
& \operatorname{Vol}(\alpha)=\sup _{\varepsilon \omega \leqslant T \in \alpha} \operatorname{Vol}(T),
\end{aligned}
$$

where the supremum is taken over all Kähler currents with analytic singularities in the class $\alpha$. By definition, the volume function is identically zero unless $X$ carries Kähler currents, and by [DP04] the latter property is equivalent to $X$ being in the Fujiki class $\mathcal{C}$ of manifolds bimeromorphic to Kähler. The results of S. Boucksom [Bou02] yield:

(1.17) Theorem ([Bou02]). If $X$ is compact complex manifold and $\alpha \in \operatorname{NS}_{\mathbb{R}}(X)$, then

$$
\operatorname{Vol}(\alpha)=\widehat{h}^{0}(X, \alpha)
$$

In other words, the growth of sections of multiples of a line bundle $L$ can be calculated as the sup of volumes of Kähler currents $T \in c_{1}(L)$ as defined above.

In section 3, we use the results of [BDPP04] and [BD09] to derive a proof of the following theorem, which, in combination with Boucksom's theorem, yields a positive answer to question (1.13) when $q=0$ and $X$ is a projective surface.

(1.18) Theorem. Let $(X, \omega)$ be a compact complex $n$-fold. Then for every class $\alpha \in H^{1,1}(X, \mathbb{R})$ we have

(a) $\operatorname{Vol}(\alpha) \leqslant \widehat{h}_{\mathrm{tr}}^{0}(X, \alpha)=\inf _{u \in \alpha} \int_{X(u, 0)} u^{n}$,

where the infimum runs over all smooth closed $(1,1)$-forms $u$ contained in the class $\alpha$.

(b) Equality holds if $X$ is a projective surface and $\alpha \in \mathrm{NS}_{\mathbb{R}}(X)$.

It would be interesting to knwow whether equality always holds without restrictions on $X$ or on $\alpha$. In the general setting of compact complex manifolds, we also hope for the following "transcendental" case of holomorphic Morse inequalities. 
(1.19) Conjecture. Let $X$ be a compact complex $n$-fold and $\alpha$ an arbitrary cohomology class in $H_{\mathrm{BC}}^{1,1}(X, \mathbb{R})$. Then

$$
\operatorname{Vol}(\alpha) \geqslant \sup _{u \in \alpha} \int_{X(u, 0) \cup X(u, 1)} u^{n}
$$

In particular, if the right hand side is positive, then $\alpha$ contains a Kähler current and $X$ must be in the Fujiki class $\mathcal{C}$.

By [Dem85], Conjecture (1.19) holds true in case $\alpha$ is an integral class. Our hope is that the general case can be attained by the diophantine approximation technique described earlier; there are however major hurdles, see [Lae02] for a few hints on these issues.

The author wishes to thank the organizers of the Riemann International School of Mathematics held in Verbania in April 2009, for the opportunity of publishing these notes in the RISM Proceedings volume.

\section{Variation of asymptotic cohomology groups}

We give here a proof a Theorem 1.8 in the context of general compact complex manifolds $X$. All norms occurring below are computed with respect to a fixed hermitian metric $\omega$ on $X$.

(2.1) Lemma. Let $X$ be a compact complex $n$-fold. Then for every coherent sheaf $\mathcal{F}$ on $X$, there is a constant $C_{\mathcal{F}}>0$ such that for every holomorphic line bundle $L$ on $X$ we have

$$
h^{q}\left(X, \mathcal{F} \otimes \mathcal{O}_{X}(L)\right) \leqslant C_{\mathcal{F}}\left(\left\|c_{1}(L)\right\|+1\right)^{p}
$$

where $p=\operatorname{dim} \operatorname{Supp} \mathcal{F}$.

Proof. We prove the result by induction on $p$; it is indeed clear for $p=0$ since we then have cohomology only in degree 0 and the dimension of $H^{0}\left(X, \mathcal{F} \otimes \mathcal{O}_{X}(L)\right)$ does not depend on $L$ when $\mathcal{F}$ has finite support. Let us consider the support $Y$ of $\mathcal{F}$ and a resolution of singularity $\mu: \widehat{Y} \rightarrow Y$ of the corresponding (reduced) analytic space. Then $\mathcal{F}$ is an $\mathcal{O}_{Y}$-module for some non necessarily reduced complex structure $\mathcal{O}_{Y}=\mathcal{O}_{X} / \mathcal{J}$ on $J$. We can look at the reduced structure $\mathcal{O}_{Y \text {,red }}=\mathcal{O}_{X} / \mathcal{J}$, $\mathcal{J}=\sqrt{\mathcal{J}}$, and filter $\mathcal{F}$ by $\mathcal{J}^{k} \mathcal{F}, k \geqslant 0$. Since $\mathcal{J}^{k} \mathcal{F} / \mathcal{J}^{k+1} \mathcal{F}$ is a coherent $\mathcal{O}_{Y \text {,red-module, }}$ we can easily reduce the situation to the case where $Y$ is reduced and $\mathcal{F}$ is an $\mathcal{O}_{Y^{-}}$ module. In that case the cohomology $H^{q}\left(X, \mathcal{F} \otimes \mathcal{O}_{X}(L)\right)=H^{q}\left(Y, \mathcal{F} \otimes \mathcal{O}_{Y}\left(L_{\mid Y}\right)\right)$ just lives on the reduced space $Y$.

Now, we have an injective sheaf morphism $\mathcal{F} \rightarrow \mu_{\star} \mu^{*} \mathcal{F}$ whose cokernel $\mathcal{G}$ has support in dimension $<p$. By induction on $p$, we conclude from the exact sequence that

$$
\left|h^{q}\left(X, \mathcal{F} \otimes \mathcal{O}_{X}(L)\right)-h^{q}\left(X, \mu_{\star} \mu^{*} \mathcal{F} \otimes \mathcal{O}_{X}(L)\right)\right| \leqslant C_{1}\left(\left\|c_{1}(L)\right\|+1\right)^{p-1}
$$


The fonctorial morphisms

$$
\begin{aligned}
& \mu^{*}: H^{q}\left(Y, \mathcal{F} \otimes \mathcal{O}_{Y}\left(L_{\mid Y}\right)\right) \rightarrow H^{q}\left(\widehat{Y}, \mu^{\star} \mathcal{F} \otimes \mathcal{O}_{\widehat{Y}}\left(\mu^{*} L\right)_{\mid Y}\right), \\
& \mu_{*}: H^{q}\left(\widehat{Y}, \mu^{\star} \mathcal{F} \otimes \mathcal{O}_{\widehat{Y}}\left(\mu^{*} L\right)_{\mid Y}\right) \rightarrow H^{q}\left(Y, \mu_{*} \mu^{\star} \mathcal{F} \otimes \mathcal{O}_{Y}\left(L_{\mid Y}\right)\right)
\end{aligned}
$$

yield a composition

$$
\mu_{*} \circ \mu^{*}: H^{q}\left(Y, \mathcal{F} \otimes \mathcal{O}_{Y}\left(L_{\mid Y}\right)\right) \rightarrow H^{q}\left(Y, \mu_{*} \mu^{\star} \mathcal{F} \otimes \mathcal{O}_{Y}\left(L_{\mid Y}\right)\right)
$$

induced by the natural injection $\mathcal{F} \rightarrow \mu_{\star} \mu^{*} \mathcal{F}$. This implies

$$
h^{q}\left(Y, \mathcal{F} \otimes \mathcal{O}_{Y}\left(L_{\mid Y}\right)\right) \leqslant h^{q}\left(\widehat{Y}, \mu^{\star} \mathcal{F} \otimes \mathcal{O}_{\widehat{Y}}\left(\mu^{*} L_{\mid Y}\right)\right)+C_{1}\left(\left\|c_{1}(L)\right\|+1\right)^{p-1} .
$$

By taking a suitable modification $\mu^{\prime}: Y^{\prime} \rightarrow Y$ of the desingularization $\widehat{Y}$, we can assume that $\left(\mu^{\prime}\right)^{*} \mathcal{F}$ is locally free modulo torsion. Then we are reduced to the case where $\mathcal{F}^{\prime}=\left(\mu^{\prime}\right)^{*} \mathcal{F}$ is a locally free sheaf on a smooth manifold $Y^{\prime}$, and $L^{\prime}=\left(\mu^{\prime}\right)^{*} L_{\mid Y}$. In this case, we apply standard analysis (e.g. [Dem85]) to conclude that $h^{q}\left(Y^{\prime}, \mathcal{F}^{\prime} \otimes \mathcal{O}_{Y^{\prime}}\left(L^{\prime}\right)\right) \leqslant C_{2}\left(\left\|c_{1}\left(L^{\prime}\right)\right\|+1\right)^{p}$. Since $\left\|c_{1}\left(L^{\prime}\right)\right\| \leqslant C_{3}\left\|c_{1}(L)\right\|$ by pulling-back, the statement follows easily.

(2.2) Corollary. For every irreducible divisor $D$ on $X$, there exists a constant $C_{D}$ such that

$$
h^{q}\left(D, \mathcal{O}_{D}\left(L_{\mid D}\right)\right) \leqslant C_{D}\left(\left\|c_{1}(L)\right\|+1\right)^{n-1}
$$

Proof. It is enough to apply Lemma 2.1 with $\mathcal{F}=\left(i_{D}\right)_{*} \mathcal{O}_{D}$ where $i_{D}: D \rightarrow X$ is the injection.

(2.3) Remark. It is very likely that one can get an "elementary" proof of Lemma 2.1 without invoking resolutions of singularities, e.g. by combining the Cartan-Serre finiteness argument along with the standard Serre-Siegel proof based ultimately on the Schwarz lemma. In this context, one would invoke $L^{2}$ estimates to get explicit bounds for the homotopy operators between Čech complexes relative to two coverings $\mathcal{U}=\left(B\left(x_{j}, r_{j}\right)\right), \mathcal{U}^{\prime}=\left(B\left(x_{j}, r_{j} / 2\right)\right)$ of $X$ by concentric balls. By exercising enough care in the estimates, it is likely that one could reach an explicit dependence $C_{D} \leqslant C^{\prime}\|D\|$ for the constant $C_{D}$ of Corollary 2.2. The proof would of course become much more technical than the rather naive brute force approach we have used.

\section{(2.4) Proof of Theorem 1.8.}

(a) We want to compare the cohomology of $L$ and $L^{\prime}=L \otimes \mathcal{O}(D)$ on $X$. For this we write $D=D_{+}-D_{-}$, and compare the cohomology of the pairs $L$ and $L_{1}=L \otimes \mathcal{O}\left(-D_{-}\right)$one one hand, and of $L^{\prime}$ and $L_{1}=L^{\prime} \otimes \mathcal{O}\left(-D_{+}\right)$on the other hand. Since $\left\|c_{1}(\mathcal{O}(D))\right\| \leqslant C\|D\|$ by elementary potential theory, we see that is 
is enough to consider the case of a negative divisor, i.e. $L^{\prime}=L \otimes \mathcal{O}(-D), D \geqslant 0$. If $D$ is an irreducible divisor, we use the exact sequence

$$
0 \rightarrow L \otimes \mathcal{O}(-D) \rightarrow L \rightarrow \mathcal{O}_{D} \otimes L_{\mid D} \rightarrow 0
$$

and conclude by Corollary 2.2 that

$$
\begin{aligned}
\left|h^{q}(X, L \otimes \mathcal{O}(-D))-h^{q}(X, L)\right| & \leqslant h^{q}\left(D, \mathcal{O}_{D} \otimes L_{\mid D}\right)+h^{q-1}\left(D, \mathcal{O}_{D} \otimes L_{\mid D}\right) \\
& \leqslant 2 C_{D}\left(\left\|c_{1}(L)\right\|+1\right)^{n-1} .
\end{aligned}
$$

For $D=\sum p_{j} D_{j} \geqslant 0$, we easily get by induction

$$
\left|h^{q}(X, L \otimes \mathcal{O}(-D))-h^{q}(X, L)\right| \leqslant 2 \sum_{j} p_{j} C_{D_{j}}\left(\left\|c_{1}(L)\right\|+\sum_{k} p_{k}\left\|\nabla_{k}\right\|+1\right)^{n-1}
$$

If we knew that $C_{D} \leqslant C^{\prime}\|D\|$ as expected in Remark 2.3, then the argument would be complete without any restriction on $D$. The trouble disappears if we fix $D$ in a finitely generated subgroup $\Gamma$ of divisors, because only finitely many irreducible components appear in that case, and so we have to deal with only finitely many constants $C_{D_{j}}$. Property (1.8 a) is proved.

(b) Fix once for all a finite set of divisors $\left(\Delta_{j}\right)_{1 \leqslant j \leqslant t}$ providing a basis of $\operatorname{DNS}_{\mathbb{R}}(X) \subset H_{\mathrm{BC}}^{1,1}(X, \mathbb{R})$. Take two elements $\alpha$ and $\beta$ in $\operatorname{DNS}_{\mathbb{R}}(X)$, and fix $\varepsilon>0$. Then $\beta-\alpha$ can be $\varepsilon$-approximated by a $\mathbb{Q}$-divisor $\sum \lambda_{j} D_{j}, \lambda_{j} \in \mathbb{Q}$, and we can find a pair $(k, L)$ with $k$ arbitrary large such that $\frac{1}{k} c_{1}(L)$ is $\varepsilon$-close to $\alpha$ and $n ! / k^{n} h^{q}(X, L)$ approaches $\widehat{h}^{q}(X, \alpha)$ by $\varepsilon$. Then $\frac{1}{k} L+\sum \lambda_{j} \Delta_{j}$ approaches $\beta$ as closely as we want. When approximating $\beta-\alpha$, we can arrange that $k \lambda_{j}$ is an integer by taking $k$ large enough. Then $\beta$ is approximated by $\frac{1}{k} c_{1}\left(L^{\prime}\right)$ with $L^{\prime}=L \otimes \mathcal{O}\left(\sum k \lambda_{j} \Delta_{j}\right)$. Property (a) implies

$$
\begin{aligned}
h^{q}\left(X, L^{\prime}\right)-h^{q}(X, L) & \geqslant-C\left(\left\|c_{1}(L)\right\|+\left\|\sum k \lambda_{j} \Delta_{j}\right\|\right)^{n-1}\left\|\sum k \lambda_{j} \Delta_{j}\right\| \\
& \geqslant-C k^{n}(\|\alpha\|+\varepsilon+\|\beta-\alpha\|+\varepsilon)^{n-1}(\|\beta-\alpha\|+\varepsilon) .
\end{aligned}
$$

We multiply the previous inequality by $n ! / k^{n}$ and get in this way

$$
\frac{n !}{k^{n}} h^{q}\left(X, L^{\prime}\right) \geqslant \widehat{h}^{q}(X, \alpha)-\varepsilon-C^{\prime}(\|\alpha\|+\|\beta\|+\varepsilon)^{n-1}(\|\beta-\alpha\|+\varepsilon) .
$$

By taking the limsup and letting $\varepsilon \rightarrow 0$, we finally obtain

$$
\widehat{h}^{q}(X, \beta)-\widehat{h}^{q}(X, \alpha) \geqslant-C^{\prime}(\|\alpha\|+\|\beta\|)^{n-1}\|\beta-\alpha\| .
$$

Property (1.8 b) follows by exchanging the roles of $\alpha$ and $\beta$. 


\section{Monge-Ampère volume formula}

The main goal of this section is to address the volume formula problem, namely whether

$$
\operatorname{Vol}(\alpha)=\inf _{u \in \alpha} \int_{X(u, 0)} u^{n} \quad(u \text { smooth })
$$

for every class $\alpha \in H_{\mathrm{BC}}^{1,1}(X, \mathbb{R})$ on a compact complex $n$-fold $(X, \omega)$.

\section{(3.2) Proof of the inequality $\leqslant$ (without restrictions)}

If $X$ does not admit any Kähler current, then the volume of every class $\alpha$ is 0 and the inequality is trivially true. Therefore we can assume that $X$ is in the Fujiki class $\mathcal{C}$. Then there exists a Kähler modification $\mu: \widetilde{X} \rightarrow X$. Assume that we have a proof for the Kähler case. Then

$$
\operatorname{Vol}(\alpha)=\operatorname{Vol}\left(\mu^{*} \alpha\right) \leqslant \inf _{v \in \mu^{*} \alpha} \int_{\widetilde{X}(v, 0)} v^{n} \leqslant \inf _{u \in \alpha} \int_{X(u, 0)} u^{n}
$$

by restricting the inf to $v=\mu^{*} u$. This shows that it is enough to consider the case when $X$ is Kähler. We have something to prove only when $\alpha \in \mathcal{E}_{X}^{+}$, i.e. when $\alpha$ contains a Kähler current (a so-called "big class"). Fix a $(1,1)$-form $u \in \alpha$. We can then introduce

$$
\varphi(x):=\sup \{\psi(x) ; \psi \leqslant 0 \text { and } u+i \partial \bar{\partial} \psi \geqslant 0 \text { on } X\}
$$

where the supremum is taken over all quasi-psh functions $\psi$ satisfying the given conditions $\psi \leqslant 0$ and $u+i \partial \bar{\partial} \psi \geqslant 0$. The following properties have been proved in [BD09] (cf. Theorem 1.4 and Corollary 2.5).

(3.4) Lemma. Let $Z_{0}$ be the analytic set of poles of any Kähler current $T_{0} \in \alpha$. Then $T=u+i \partial \bar{\partial} \varphi \geqslant 0$ and $\varphi$ is continuous with locally bounded second derivatives $\partial^{2} / \partial z_{j} \partial \bar{z}_{k}$ on $X \backslash Z_{0}$. Moreover, if $S$ is the set of points $z \in X \backslash Z_{0}$ where $\varphi(z)=0$, then $S \subset\{z ; u(z) \geqslant 0\}$ and

$$
\operatorname{Vol}(\alpha)=\int_{S} u^{n}=\int_{X \backslash Z_{0}}(u+i \partial \bar{\partial} \varphi)^{n}
$$

Since $S \subset\{z ; u(z) \geqslant 0\}$, we immediately conclude from these equalities that

$$
\operatorname{Vol}(\alpha) \leqslant \int_{\{z ; u(z) \geqslant 0\}} u^{n}=\int_{\{z ; u(z)>0\}} u^{n}=\int_{X(u, 0)} u^{n} .
$$

(3.5) Proof of the volume formula for $\alpha \in \mathrm{NS}_{\mathbb{R}}(X)$ on a projective surface.

By definition, the volume $\operatorname{Vol}(\alpha)$ is obtained as the supremum of $\int_{X \backslash \operatorname{sing}(T)} T^{n}$ for Kähler currents with analytic singularities in $\alpha$. By [Dem92] and [BDPP04], 
there exists a blow-up $\mu: \widetilde{X} \rightarrow X$ such that $\mu^{*} T=[E]+\beta$ where $E$ is a normal crossing divisor on $\widetilde{X}$ and $\beta \geqslant 0$ smooth. Until now, this is valid for an arbitrary compact complex manifold $X$. If moreover $X$ is projective and $\alpha \in \mathrm{NS}_{\mathbb{R}}(X)$, it is shown in [BDPP04] that we have the "orthogonality property"

$$
[E] \cdot \beta^{n-1}=\int_{E} \beta^{n-1} \leqslant C\left(\operatorname{Vol}(\alpha)-\beta^{n}\right)^{1 / 2},
$$

in other words, $E$ and $\beta$ become "more and more orthogonal" as $\beta^{n}$ approaches the volume. Our method consists of approaching $[E]+\beta$ by smooth closed $(1,1)$-forms $u_{\varepsilon}$ in the same $\partial \bar{\partial}$-cohomology class as $[E]+\beta$, in such a way that

$$
\int_{\widetilde{X}\left(u_{\varepsilon}, 0\right)} u_{\varepsilon}^{n}
$$

will not be substantially larger than the volume $\int_{\widetilde{X}} \beta^{n}$. For this, we select a hermitian metric $h$ on $\mathcal{O}(E)$ and put

$$
u_{\varepsilon}=\frac{i}{2 \pi} \partial \bar{\partial} \log \left(\left|\sigma_{E}\right|_{h}^{2}+\varepsilon^{2}\right)+\Theta_{\mathcal{O}(E), h}+\beta
$$

where $\sigma_{E} \in H^{0}(\widetilde{X}, \mathcal{O}(E))$ is the canonical section and $\Theta_{\mathcal{O}(E), h}$ the Chern curvature form. Clearly,by the Lelong-Poincaré equation, $u_{\varepsilon}$ converges to $[E]+\beta$ in the weak topology as $\varepsilon \rightarrow 0$. Straightforward calculations yield

$$
u_{\varepsilon}=\frac{i}{2 \pi} \frac{\varepsilon^{2} D_{h}^{1,0} \sigma_{E} \wedge \overline{D_{h}^{1,0} \sigma_{E}}}{\left(\varepsilon^{2}+\left|\sigma_{E}\right|^{2}\right)^{2}}+\frac{\varepsilon^{2}}{\varepsilon^{2}+\left|\sigma_{E}\right|^{2}} \Theta_{E, h}+\beta .
$$

The first term converges to $[E]$ in the weak topology, while the second, which is close to $\Theta_{E, h}$ near $E$, converges pointwise everywhere to 0 on $\widetilde{X} \backslash E$. A simple asymptotic analysis shows that

$$
\left(\frac{i}{2 \pi} \frac{\varepsilon^{2} D_{h}^{1,0} \sigma_{E} \wedge \overline{D_{h}^{1,0} \sigma_{E}}}{\left(\varepsilon^{2}+\left|\sigma_{E}\right|^{2}\right)^{2}}+\frac{\varepsilon^{2}}{\varepsilon^{2}+\left|\sigma_{E}\right|^{2}} \Theta_{E, h}\right)^{p} \rightarrow[E] \wedge \Theta_{E, h}^{p-1}
$$

in the weak topology for $p \geqslant 1$, hence

$$
\lim _{\varepsilon \rightarrow 0} u_{\varepsilon}^{n}=\beta^{n}+\sum_{p=1}^{n}\left(\begin{array}{l}
n \\
p
\end{array}\right)[E] \wedge \Theta_{E, h}^{p-1} \wedge \beta^{n-p} .
$$

In arbitrary dimension, the signature of $u_{\varepsilon}$ is hard to evaluate, and it is also non trivial to decide the sign of the limiting measure $\lim u_{\varepsilon}^{n}$. However, when $n=2$, we get the simpler formula

$$
\lim _{\varepsilon \rightarrow 0} u_{\varepsilon}^{2}=\beta^{2}+2[E] \wedge \beta+[E] \wedge \Theta_{E, h}
$$


In this case, $E$ can be assumed to be an exceptional divisor (otherwise some part of it would be nef and could be removed from the poles of $T$ ). Hence the matrix $\left(E_{j} \cdot E_{k}\right)$ is negative definite and we can find a hermitian metric $h$ on $\mathcal{O}(E)$ such that $\left(\Theta_{E, h}\right)_{\mid E}<0$. Then $[E] \wedge \Theta_{E, h}$, which is the limit of the product of the first two terms in $u_{\varepsilon}^{2}$, contributes negatively to the limit; all other terms are nonnegative or have a mass converging to 0. From this, one can easily infer by (3.6) that

$$
\limsup _{\varepsilon \rightarrow 0} \int_{\widetilde{X}\left(u_{\varepsilon}, 0\right)} u_{\varepsilon}^{2} \leqslant \int_{\widetilde{X}} \beta^{2}+2[E] \wedge \beta \leqslant \operatorname{Vol}(\alpha)+2 C\left(\operatorname{Vol}(\alpha)-\beta^{2}\right)^{1 / 2} .
$$

This is arbitrary close to $\operatorname{Vol}(\alpha)$ when $\beta^{2}$ approaches the volume, and so property $(1.18 \mathrm{~b})$ is proved in dimension 2 . Obviously the $n$-dimensional case would require a deeper analysis of "higher order" orthogonality relations.

\section{References}

[AG62] Andreotti, A., Grauert, H.: Théorèmes de finitude pour la cohomologie des espaces complexes; Bull. Soc. Math. France 90 (1962) 193-259.

[BL04] Birkenhake, Ch., Lange, H.: Complex Abelian Varieties; Second augmented edition, Grundlehren der Math. Wissenschaften, Springer, Heidelberg, 2004.

[Bou02] Boucksom, S.: On the volume of a line bundle; Internat. J. Math. 13 (2002), 10431063.

[BD09] Berman, R., Demailly, J.-P.: Regularity of plurisubharmonic upper envelopes in big cohomology classes; arXiv: math.CV/0905.1246, to appear in the Proceedings of the volume "Geometry and Topology" in honor of Oleg Y. Viro, edited by B. Juhl-Jöricke and M. Passare, Birkhaüser.

[BDPP04] Boucksom, S., Demailly, J.-P., Păun, M., Peternell, Th.: The pseudo-effective cone of a compact Kähler manifold and varieties of negative Kodaira dimension; arXiv: math.AG/0405285, see also Proceedings of the ICM 2006 in Madrid.

[Dem85] Demailly, J.-P.: Champs magnétiques et inégalités de Morse pour la d" -cohomologie; Ann. Inst. Fourier (Grenoble) 35 (1985), 189-229.

[Dem91] Demailly, J.-P.: Holomorphic Morse inequalities; Lectures given at the AMS Summer Institute on Complex Analysis held in Santa Cruz, July 1989, Proceedings of Symposia in Pure Mathematics, Vol. 52, Part 2 (1991), 93-114.

[Dem90] Demailly, J.-P.: Singular hermitian metrics on positive line bundles; Proceedings of the Bayreuth conference "Complex algebraic varieties", April 2-6, 1990, edited by K. Hulek, T. Peternell, M. Schneider, F. Schreyer, Lecture Notes in Math. n 1507 , Springer-Verlag, 1992.

[Dem92] Demailly, J.-P.: Regularization of closed positive currents and Intersection Theory; J. Alg. Geom. 1 (1992), 361-409.

[DEL00] Demailly, J.-P., Ein, L., and Lazarsfeld, R.: A subadditivity property of multiplier ideals; Michigan Math. J. 48 (2000), 137-156.

[DP04] Demailly, J.-P., Păun, M: Numerical characterization of the Kähler cone of a compact Kähler manifold; arXiv: math.AG/0105176; Annals of Math. 159 (2004) 1247-1274.

[FKL07] de Fernex, T., Küronya, A., Lazarsfeld, R.: Higher cohomology of divisors on a projective variety; Math. Ann. 337 (2007) 443-455.

[Fuj94] Fujita, T.: Approximating Zariski decomposition of big line bundles; Kodai Math. J. 17 (1994) 1-3. 
14 Asymptotic cohomological inequalities: a tribute to Riemann

[Hir54] Hirzebruch, F.: Arithmetic genera and the theorem of Riemann-Roch for algebraic varieties; Proc. Nat. Acad. Sci. U.S.A. 40 (1954), 110-114.

[Hir56] Hirzebruch, F.: Neue topologische Methoden in der algebraischen Geometrie; Ergebnisse der Mathematik und ihrer Grenzgebiete (N.F.), Heft 9. Springer-Verlag, Berlin-Göttingen-Heidelberg, 1956.165 pp; English translation: Topological methods in algebraic geometry; Springer-Verlag, Berlin (1966).

[Kur06] Küronya, A.: Asymptotic cohomological functions on projective varieties; Amer. J. Math. 128 (2006) 1475-1519.

[Lae02] Laeng, L.: Estimations spectrales asymptotiques en géométrie hermitienne; Thèse de Doctorat de l'Université de Grenoble I, octobre 2002, http://www-fourier.ujf-grenoble.fr/THESE/ps/laeng.ps.gz and http://tel.archives-ouvertes.fr/tel-00002098/en/.

[Laz04] Lazarsfeld, R.: Positivity in Algebraic Geometry I.-II; Ergebnisse der Mathematik und ihrer Grenzgebiete, Vols. 48-49., Springer Verlag, Berlin, 2004.

[Rie57] Riemann, B.: Theorie der Abel'schen Functionen; J. für Math. 54 (1857), in Gesammelte mathematische Werke (1990) 120-144.

[Roc65] Roch, G.: Über die Anzahl der willkurlichen Constanten in algebraischen Functionen; J. für Math. 64 (1865) 372-376.

(version of February 23, 2010, printed on October 4, 2018) 